\title{
Progress, Opportunities and Challenges in Modeling of Plasma Etching
}

\author{
Yang Yang ${ }^{1}$, Mingmei Wang ${ }^{2}$ and Mark J. Kushner ${ }^{1}$ \\ ${ }^{1}$ Department of Electrical and Computer Engineering \\ ${ }^{2}$ Department of Chemical and Biological Engineering \\ Iowa State University, 104 Marston Hall, Ames, IA 50010 USA \\ yangying@iastate.edu,mmwang@iastate.edu, mjk@iastate.edu
}

\begin{abstract}
The development of plasma etching processes is challenged by the complexity of the chemistries and the unpredictability of the performance of plasma tools due to sometimes subtle changes in design. Challenges also face the use of modeling and simulation to provide a computational assist to the development of these processes. Selected issues in developing this modeling capability will be discussed.
\end{abstract}

\section{Introduction}

The use of modeling and simulation for the development of plasma equipment and etch processes has made significant progress over the past decade. Reactor scale modeling tools linked to profile simulators are now actively used in the design of physical vapor deposition, plasma deposition, plasma etching and ion implantation tools [1,2]. These modeling efforts have had quantifiable improvements in the development cycle of these tools as well as more qualitative improvements in our fundamental understanding of the plasma transport and chemistry occurring in the tools. Although these successes are encouraging, there are still significant challenges that face modeling and simulation to continue to provide added value to the semiconductor fabrication industry.

The origins of these challenges include the introduction of new materials (e.g., high-k dielectrics) for which knowledge bases are limited, increasing the dynamic range of operating conditions (e.g., very high frequency plasma sources) that extend beyond the realm of established modeling techniques and accurately addressing manufacturing issues that require extreme spatial resolution. Addressing these challenges will require, as a highest priority, an improvement in the knowledge base of material properties for plasma transport (e.g., cross sections and transport coefficients) but, more importantly, for the plasma surface interactions that result in feature evolution. Beyond those improvements in knowledge bases lie challenges in creating more robust modeling platforms that are able to predict new operating regimes, as opposed to simply analyzing those initially empirically investigated.

In this paper, we will discuss a selection of challenges facing plasma equipment and process modeling from perspective of feature evolution and advanced reactor concepts.

\section{Description of Models}

The models used in our investigations are the HPEM, and MCPFM. The Hybrid Plasma Equipment Model is a 2dimensional hybrid simulator in which the densities, fluxes and temperatures of all charged and neutral species are solved for on a rectilinear mesh [3]. Solutions of Maxwell's and
Poisson's equations are included, as are kinetic simulations of electron and ion transport. The Monte Carlo Feature Profile Model uses fluxes from the HPEM to predict the evolution of surface features [4].

\section{Select Challenges for the Modeling Infrastructure}

\section{Advance Reactor Concept: Very High Frequency Power}

An advanced feature of RIE plasma tools currently under development and deployment is use of very high frequency power sources (> $100 \mathrm{MHz}$ ) [5]. The goal of this strategy is to better control the resulting electron energy distributions in the plasma and so control the cracking patterns of the feedstock gases by electron impact dissociation. This will produce finer control over the reactant fluxes to the substrate. As the plasma reduced wavelength of the rf power applied to the reactor approaches that of the size of the reactor, finite wavelength effects become increasingly more important. This in turn becomes increasingly more challenging for modeling due to the need for including a full solution of the electromagnetic Maxwell's equations, as opposed to only the electrostatic Poisson's equation. These approaches should resolve simultaneous capacitive and inductive coupling.

Operating in this high frequency regime is particularly sensitive to the details of the design of the reactor such as where the rf power is applied to the reactor and the path the electromagnetic wave follows from the power cable to the plasma. To address these issues, a full, time-domain Maxwell equation solver was implemented into the HPEM with the ability to specify the location that power is fed into the reactor. The geometry and material properties between the cable and the absorption of that power in the plasma, in addition to the properties of the plasma itself, then determine the spatial distribution the plasma.

An example of the consequences of high frequency operation of RIE tools is shown in Figs. 1 and 2. Rf power is fed into the reactor on axis at the top of the reactor. The top electrode is encased in a dielectric. The bottom electrode is grounded for this example. A plasma in argon at 50 mTorr is sustained at $300 \mathrm{~W}$. As the frequency of excitation increases, from $10 \mathrm{MHz}$ to $160 \mathrm{MHz}$, the incident power transitions from being largely electrostatically coupled to being largely electromagnetically coupled. This is manifested by a change in the distribution in power from being edge peaked at low frequencies to being center peaked at high frequencies. As the power is deposited in a more wave-like manner, there is constructive interference in the waves in the center of the reactor, which peaks both power deposition and plasma density. The center peaking does not occur when including only electrostatic coupling. This transition is evident in there being a radially dependent phase change in the electric field in the sheath of the powered electrode. Small changes in the 
conductivity for the plasma, intervening materials and the location of the power feed into the reactor can have significant influence on the distribution of power.
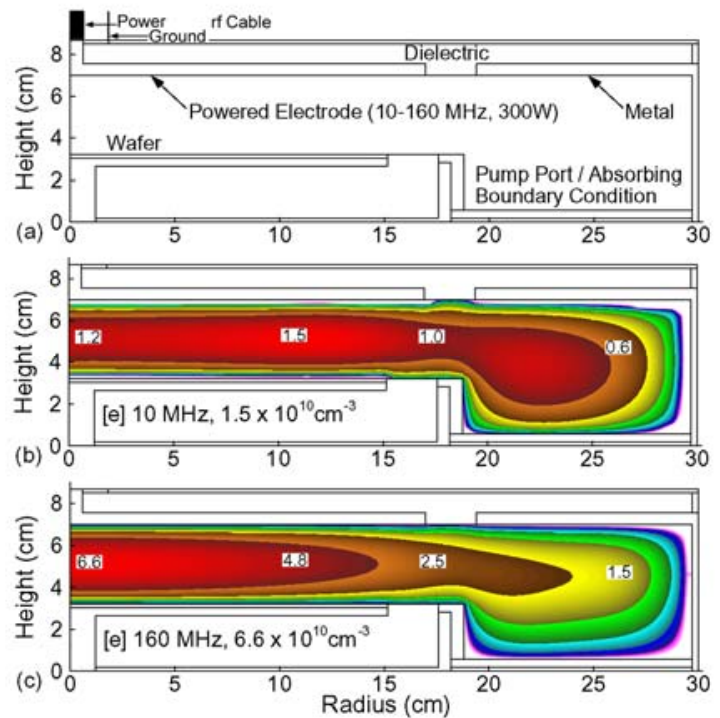

Fig. 1 - Modeling of very high frequency RIE plasma tools. (a) Schematic showing the location of the rf power feed and intervening materials. (b) Plasma density for $10 \mathrm{MHz}, \mathrm{Ar}, 50 \mathrm{mT}$ Torr and $300 \mathrm{~W}$. Maximum density is noted. (c) Density for $160 \mathrm{MHz}$.
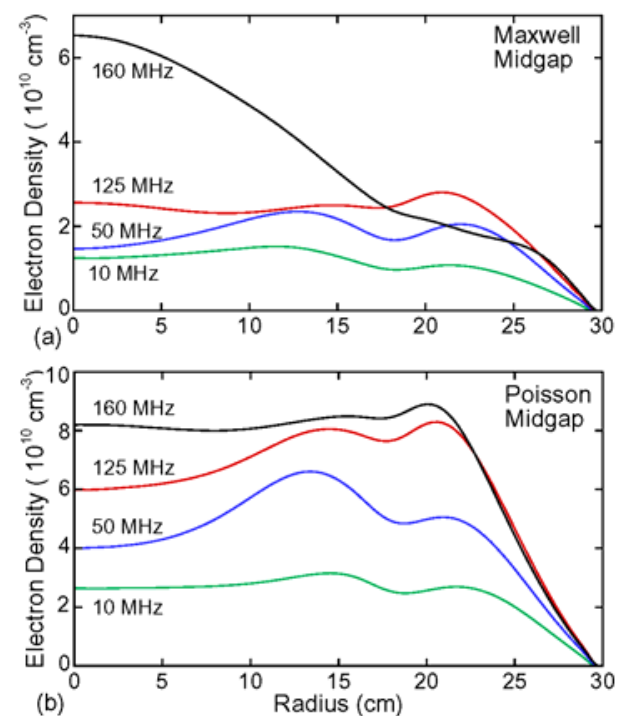

Fig. 2 - Plasma density at mid-gap for the RIE tool (Ar, 50 mTorr, $300 \mathrm{~W}$ ) while varying the rf frequency. (a) Full wave time-domain Maxwell equation solver. (b) Electrostatic Poisson equation solver.

\section{Charging, Stochastic and Errant Behavior}

The plasma etching of extremely high aspect ratio (HAR) features (aspect ratio $>50-100$ ) is challenging from many perspectives. In particular, maintaining the critical dimension of such features is challenged by errant behavior. This is where the vast majority of, for example, contacts are straight with well maintained CDs while there is an occasional (perhaps as few as $1 \%$ ) of the contacts displaying twisting where the contact will swerve to one side. At first examination, these errant features should not occur because over the tens or hundreds of microns over which these errant features occur, there is for all practical purposes no change in any measurable plasma property.
This errant behavior may be attributed to either or both of statistical variations in fluxes of reactants entering into the opening of the contact or charging. The statistical variation results from the finite fluxes and small opening size. Many of these etching processes use fluorocarbon gas mixtures which rely on the co-deposition of polymers to both achieve selectivity and to provide the precursors for etching. As such, there are at least four classes of reactants entering the contact: ions, electrons, polymerizing radicals and etching radicals. When contact sizes are only tens to $100 \mathrm{~nm}$ in diameter, there is a statistical fluctuation in the identity and order of these precursors into the feature. The order of the reactants entering the feature producing polymerization, etching or charging is important to the evolution of the profile.

Modeling this errant behavior is also very challenging from at least two perspectives, particularly on the feature scale. First, due to the dynamics of the charging and etch processes, the errant behavior can only be accurately represented with atomic resolution. Second, the low rate of occurrence of the twisting requires very large numbers of cases to be run to reproduce it.

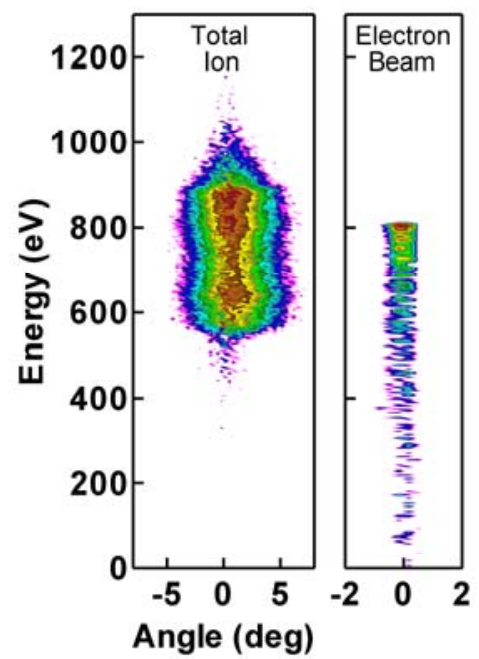

Fig. 3 - Charged particle energy and angular distributions to the wafer. (left) Total ion flux. (right) High energy electron beam flux.

The results from HPEM and MCFPM have been combined to investigate the consequences of stochastic random fluxes into small features on twisting. The computational compromise we made to address these issues is to address small enough feature so that the mesh can resolve single atoms while assuming that the aspect ratio is the more important parameter. A capacitively coupled plasma tool similar to that in Fig. 1 was modeled using an $\mathrm{Ar} / \mathrm{C}_{4} \mathrm{~F}_{8} / \mathrm{O}_{2}=$ $80 / 15 / 5$ mixture at $40 \mathrm{mTorr}$ and $1 \mathrm{~kW}$ at $10 \mathrm{MHz}$ on the lower electrode. The resulting cycle averaged ion energy and angular distribution (sum of all ions) is shown in Fig. 3. The HAR features etched into $\mathrm{SiO}_{2}$ using a hard mask are shown in Fig. 4. Charging and subsequent generation of electric fields inside the feature were included. The modeling of the sequence of features is the same except that a different random number generator seed was used for the Monte Carlo simulation for each case. The twisting and case-to-case change in the profile results solely from the randomness of the fluxes into the feature. Note that selected cases were chosen to illustrate the errant behavior (many more cases have straight profiles) although the small size of the feature exaggerates the errant behavior. 

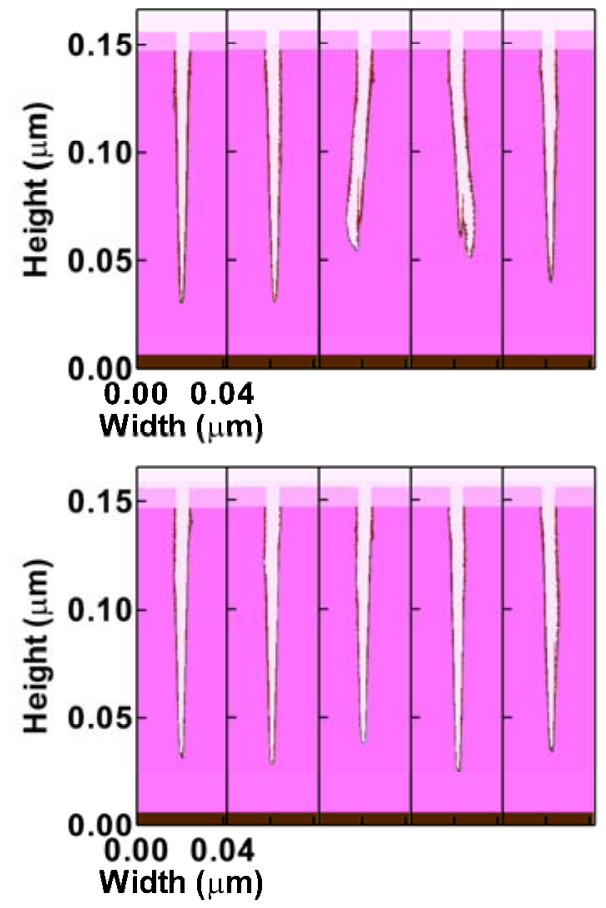

Fig. 4 - Predictions of HAR profiles etched in $\mathrm{SiO}_{2}$ for small features where stochastic processes are exaggerated. (top) Without electron beam fluxes. (bottom) With electron beam fluxes. The profiles are for otherwise the same conditions except for the choice of random number in the Monte Carlo simulation.

\section{Advanced Reactor Concepts: DC Augmented RIE}

Improving the rate of development of plasma equipment and processes requires a robust modeling platform that is able to capture advanced concepts while not changing the previously used algorithms. That is, if unique algorithms are employed to address specific tool components, it is unclear if predicted changes in performance with respect to baseline designs is due to the change algorithm or change in design. As such, modeling is highly challenged to keep the algorithms as fundamental as possible to capture as broad a technology operating regime as possible while not being computationally over-burdensome.

An example of this challenge is including new power sources in a self-consistent manner. One such plasma tool uses rf plasma sources which are augmented with an externally applied negative dc bias on the electrode opposite to the wafer [6]. The intent is to provide additional control over ionization while also generating a beam of high energy, narrow angle electrons which are able to penetrate into features and counter the possible deleterious effects of charging by positive ions.

A dc-augmented power source was incorporated into the HPEM as a self-consistent add-on to the baseline rf algorithms. In doing, so, the consequences of the dcaugmented power on the rf and self-generated dc biases on the substrate to deliver a specified power were accounted for, as well as the net dc current flowing through the reactor. The plasma potential as a function of height during the rf cycle for a $200 \mathrm{~W}$ dc power source added to the reactor discussed above is shown in Fig. 5. The electron energy and angular distribution of the beam electrons incident onto the wafer are shown in Fig. 3. The conditions are the same as discussed for HAR etching. At any given instant during the rf cycle, the maximum possible electron energy incident onto the wafer is the difference in the dc potential on the upper electrode and the phase dependent potential on the lower electrode. This produces a broad range of electron energies with a narrow angular spread. Collisions of the electrons in transit across the plasma further spread their energies.

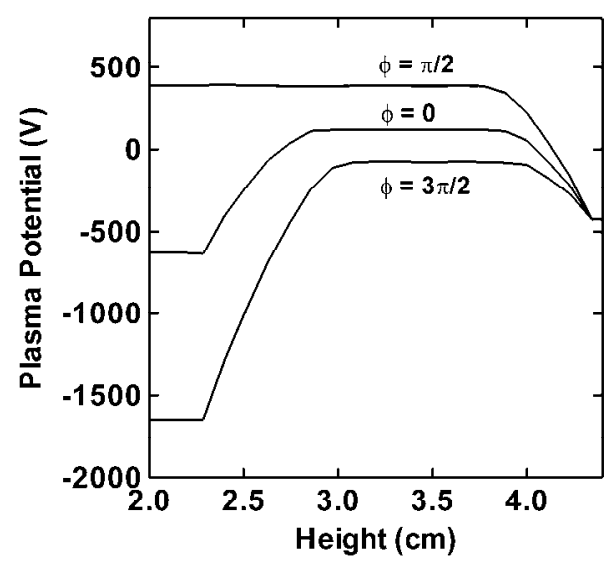

Fig. 5 - Plasma potential as a function of height and phase during the rf cycle. The rf biased wafer is at the left. The dc biased top electrode is on the right.

The resulting HAR features etched into $\mathrm{SiO}_{2}$ are shown in Fig. 4. Note that the errant twisting of the features is largely eliminated by the dc-augmented power producing the narrow angle, high energy electron beam. This is due to the elimination of local extremes in positive electric potential resulting from stochastically arriving ions able to penetrate deep into the feature. There is, however, still a significant feature-to-feature variation in etch rate. This results from the randomness of the neutral fluxes and the activation energy provided by ions.

\section{Concluding Remarks}

The opportunities and challenges to improve process development by modeling will ultimately be met when plasma and profile models are adaptive, self-aware and selfcorrecting to the changing conditions in the reactor. Models which automatically generate their own reaction mechanisms and reaction rate coefficients, and have access to databases that are automatically queried will be required to not only keep up with but lead the industry in the development of advanced plasma chemistries.

\section{Acknowledgments}

This work was supported by the Semiconductor Research Corp., TEL Inc., Micron Inc. and Applied Materials, Inc.

\section{References}

[1] P.L.G. Ventzek, S. Rauf, P.J. Stout, D. Zhang, W. Dauksher and E. Hall, Appl. Surf. Sci. 192, 201 (2002).

[2] S. Rauf, L. A. Gochberg, P. L.G. Ventzek and E. J. McInerney, Semiconductor International, Nov. 2005.

[3] M. J. Kushner, J. Appl. Phys. 94, 1436 (2003).

[4] A. Sankaran and M. J. Kushner J. Appl. Phys. 97, 023307 (2005).

[5] G. A. Hebner, E. V Barnat, P. A Miller, A. M. Paterson and J. P. Holland, Plasma Source Sci. Technol. 15, 879 (2006).

[6] Tokyo Electron, Ltd., http://www.tel.com 